\title{
Dutch Books and nonclassical probability spaces
}

\author{
Leszek Wroński ${ }^{1} \cdot$ Michał Tomasz Godziszewski $^{2}$
}

Received: 25 November 2015 / Accepted: 7 July 2016 / Published online: 29 July 2016 (C) The Author(s) 2016. This article is published with open access at Springerlink.com

\begin{abstract}
We investigate how Dutch Book considerations can be conducted in the context of two classes of nonclassical probability spaces used in philosophy of physics. In particular we show that a recent proposal by B. Feintzeig to find so called "generalized probability spaces" which would not be susceptible to a Dutch Book and would not possess a classical extension is doomed to fail. Noting that the particular notion of a nonclassical probability space used by Feintzeig is not the most common employed in philosophy of physics, and that his usage of the "classical" Dutch Book concept is not appropriate in "nonclassical" contexts, we then argue that if we switch to the more frequently used formalism and use the correct notion of a Dutch Book, then all probability spaces are not susceptible to a Dutch Book. We also settle a hypothesis regarding the existence of classical extensions of a class of generalized probability spaces.
\end{abstract}

Keywords Generalized probability spaces · Dutch Books · Nonclassical probability

\section{Introduction}

It seems reasonable to say that the fundamentals of quantum mechanics, and in particular the need to consider incompatible measurements, i.e. measurements which cannot be simultaneously conducted, suggest the employment of some notion of

Leszek Wroński

leszek.wronski@uj.edu.pl

1 Jagiellonian University, Kraków, Poland

2 University of Warsaw, Warsaw, Poland 
nonclassical probability spaces. A natural hope would be that these spaces would not be marked by irrationality in the form of being prone to a Dutch Book. However, it is nontrivial how betting should be understood in nonclassical environments. In that context a recent paper (Feintzeig 2015) sketches an interesting research goal. It is well known that classical probability spaces are not susceptible to a Dutch Book-in fact, a function associating real numbers to elements of a Boolean algebra is a classical probability function if and only if no Dutch Book, that is, a set of bets with guaranteed non-zero loss, can be constructed using it (we will discuss this in detail in the next section; for a helpful introduction see Vineberg (2011), and for a formal proof see Kemeny 1955). This can be used (see e.g. section 3.2.2. of Bradley (2015) or chapter 5.3. of Christensen 2004) as a basis for arguments for "probabilism"-a norm dictating that a rational agent's degrees of belief should be classically conceived probabilities.

It is interesting to consider cases in which the objects of an agent's beliefs do not form a Boolean algebra, which typically happens e.g. in quantum contexts. ${ }^{1}$ One way this can be formally modelled is by using "generalized probability spaces" which do not have an "extension" in the form of a classical probability space: that is, they are not classical "in disguise", but are inherently non-classical. (We will discuss below both the notion of "extension" and how the fact that a space possesses a classical extension is connected with its not describing any inherently nonclassical phenomena.) Feintzeig proposes that, in addition to this, we should look for generalized probability spaces which are not susceptible to a Dutch Book, which would be a mark of epistemic irrationality. We will show here that these two goals, that is, not possessing a classical extension and not being susceptible to a Dutch Book, cannot be simultaneously achieved, at least among finite structures. We prove, using the particular notions employed in Feintzeig (2015), that a finite generalized probability space does not have a classical extension if and only if it is susceptible to a Dutch Book.

This does not mean that employing any notion of a non-classical generalized probability space shall be avoided in philosophical analysis of quantum phenomena due to the above-mentioned mark of irrationality. We will argue that the classical Dutch Book notion cannot be put to use without any changes, if one wants to argue against using a given non-classical generalized probability function. We will point out that the literature on "quantum bayesianism"2 contains a well-established way of thinking about betting, but it is employed in the context of different sort of nonclassical probability spaces than the ones used by Feintzeig. Those spaces are directly motivated by quantum mechanical structures and could be labelled as "lattice-theoretic". We point out that in the context of lattice-theoretic spaces a part of Feintzeig's project is trivially satisfied: no lattice-theoretic nonclassical probability space is susceptible to a Dutch Book. This is an indirect and of course inconclusive argument in favour of using the lattice-theoretic notion of a nonclassical probability space.

The format of the paper is as follows. In the next section we will rehearse the classical Dutch Book argument and hint at its aspects which might be troublesome

\footnotetext{
${ }^{1}$ But not only there-see e.g. section IIIB.3 in Fine (1973).

${ }^{2}$ Some authors in that field, e.g. Fuchs, Schack and Mermin, now refer to the view as "QBism".
} 
in nonclassical cases. In Section 3 we show that Feintzeig's project is unrealisable using the notions employed in his paper, and also settle his hypothesis regarding the existence of classical extensions of generalized probability spaces satisfying the condition of finite subadditivity. The status of the project if we switch to the latticetheoretic spaces and use a notion of a Dutch Book suitable for nonclassical contexts is discussed in Section 4. We will be defining all notions as we go. Proofs of the results and mathematical details of the examples are relegated to the Appendix. If not specified otherwise, the structures we are talking about are finite, like in Feintzeig's paper.

\section{The structure of the classical Dutch Book argument}

As already mentioned, it is well known that a function associating real numbers to elements of a Boolean algebra is a classical probability function if and only if it cannot be used to construct a set of bets with a guaranteed non-zero loss on part of one of the gamblers (see Kemeny 1955). This has epistemological consequences if we think of the function as specifying some agent's betting quotients. An agent's betting quotient for $A$ is $q$ when $q S$ is the price of a bet which pays $S$ out of the sellers pocket if $A$ is true (and nothing otherwise) which the agent would choose if she knew she was to take part in that bet, but did not know whether she would be buying or selling, and wanted to avoid loss. According to many authors this means that a bet priced as such is a "fair bet" according to that agent, which might be a slightly unorthodox, if not outright Pickwickian, usage of the term "fair", but one which we will adopt anyway. The agent's degrees of belief are then, in a move which goes back to Ramsey (1931), identified with her betting quotients. If, then, elements of a Boolean algebra are the propositions ${ }^{3}$ in which the agent holds some degree of belief, and the function associating real numbers with those propositions specifies her betting quotients, then the existence of a Dutch Book supposedly points to some irrationality on part of the agent: after all, all the bets comprising the Dutch Book are fair by her lights, and all the same she can see the whole set of bets taken together guarantees her loss. Something has to be wrong with her degrees of belief. This the core of the classical Dutch Book argument for probabilism.

A key aspect of that argument, and it would seem of any argument using the notion of a Dutch Book, is that the loss on part of the agent is not merely expected, but guaranteed. She ends up losing money whatever happens. How do we check if that is the case? Typically we go through some set of pairwise incompatible but jointly exhaustive propositions in the algebra, consider each of them in turn as true, and calculate the results of all the bets. For example, if we want to exploit the fact that the agent violates additivity, that is, for some incompatible propositions $A$ and $B$ her

\footnotetext{
${ }^{3}$ We will use the terms "propositions" and "events" interchengeably, since our topic is neither ontology nor philosophy of language. Would someone need an intended interpretation, please think of both as sets of possible worlds.
} 
degree of belief in $A \vee B$ is not equal to the sum of her degrees of belief in $A$ and in $B$, we consider three bets: regarding $A$, regarding $B$, and regarding $A \vee B$. (Who is buying and who is selling each bet depends on the direction in which the agent violates additivity.) We then check the agent's gain in three pairwise incompatible but jointly exhaustive cases: $A \wedge \neg B, \neg A \wedge B$, and $\neg A \wedge \neg B$. If we set up the Dutch Book correctly, we see that in each of the cases the agent ends up losing money.

Frequently, the set of propositions in which the agent holds a degree of belief is given as a set of subsets of the same "base" set $W$, which can be variously understood, e.g. as containing atomic outcomes of some experiment, or worlds that are epistemically possible for the agent. In that case we can go through each element $w \in W$, see which propositions end up true if $w$ "is the case", that is, for which propositions $A$ it is true that $w \in A$, and calculate the winnings accordingly. This is simply a generalisation of the above procedure, and is the way Feintzeig proceeds in his paper.

The above amounts to a definition of a Dutch Book that encompasses both the classical notion and one usable in the context of generalized probability spaces:

Definition 1 (Dutch Book) Let $W$ be a non-empty set and let $\mathcal{F} \subseteq \mathcal{P}(W)$ be a non-empty family of its subsets such that $\bigcup \mathcal{F} \neq \varnothing$. Let $q: \mathcal{F} \rightarrow \mathbb{R}$ be a real-valued function. We say that $q$ is susceptible to a Dutch Book (is DutchBookable; permits a Dutch Book) if there exists a function $s: \mathcal{F} \rightarrow \mathbb{R}$ and $\mathcal{F}_{0}$, a finite non-empty subset of $\mathcal{F}$, such that for any $w \in \cup \mathcal{F}$ the following inequality holds:

$$
U(w)=\sum_{E \in \mathcal{F}_{0}: w \in E}(1-q(E)) s(E)-\sum_{E \in \mathcal{F}_{0}: w \notin E} q(E) s(E)=\sum_{E \in \mathcal{F}_{0}}\left(\chi_{E}(w)-q(E)\right) s(E)<0
$$

where $\chi_{E}$ is the characteristic function of the set $E$.

We can also say in such a case that the space $\langle W, \mathcal{F}, q\rangle$ permits a Dutch Book or that an epistemic agent with a credence function $q$ is susceptible to a Dutch Book.

The set $W$ may be thought of as a sample space, a class of possible worlds etc. The condition $\bigcup \mathcal{F} \neq \varnothing$ guarantees that the family $\mathcal{F}$ does not consist solely of the empty subset of $W$. When $\mathcal{F}$ is a finite Boolean algebra, the condition that the inequality $U(w)<0$ holds for any $w \in \bigcup \mathcal{F}$ amounts then to saying that it holds for all elements of all atoms of the algebra $\mathcal{F}$.

The motivation for such a definition is that it captures the properties of Dutch Books with some generality. Usually, $q$ represents betting quotients (which correspond to credences of an epistemic agent), i.e. measures of chances of events in $\mathcal{F}$ that an agent is willing to accept as fair quotients for a bet about those events. The numbers $s(E)$ represent the stakes of bets regarding the respective events.

We would like to suggest the following justification for the formula used in the above definition of a Dutch Book. For any $E \in \mathcal{F}_{0}$, if $s(E) \geq 0$, then we say that the bet is bought by the agent, i.e. it is a bet for the event $E$. A bet for $E \operatorname{costs} q(E) s(E)$. If the agent wins this bet (i.e. if $w \in E$ ), she receives $s(E)$, and otherwise she receives 
nothing. Therefore her profit from all bets for events in $\mathcal{F}_{0}$ with $s(E) \geq 0$ is then equal to:

$$
\sum_{E \in \mathcal{F}_{0}: s(E) \geq 0}\left(\sum_{w: w \in E}(1-q(E)) s(E)+\sum_{w: w \notin E}-q(E) s(E)\right) .
$$

Analogously, if $s(E)<0$, then we say that the bet is sold by the agent, i.e. it is a bet against the event $E$. For a bet against $E$ the agent receives $-q(E) s(E)$ (observe that this is a positive number for $q(E) \geq 0$ ). If she loses this bet (i.e. if $w \in E$ ) she has to pay $-s(E)$, while otherwise she keeps the $-q(E) s(E)$. Therefore her profit from all bets for all events in $\mathcal{F}_{0}$ with $s(E)<0$ is then equal to:

$$
\sum_{E \in \mathcal{F}_{0}: s(E)<0}\left(\sum_{w: w \in E}(1-q(E)) s(E)+\sum_{w: w \notin E}-q(E) s(E)\right) .
$$

When we combine the two formulas, taking the sum over the entire $\mathcal{F}_{0}$, we obtain the formula from the definition of a Dutch Book.

We suggest that the Readers who would like a further justification of the formal details of the above definition read the next part of the text, rendered in smaller print. The main thread of the paper continues in regularly sized print soon thereafter.

In the classical case, when $W$ is finite and $\mathcal{F}$ is interpreted as a finite algebra of events, $\mathcal{F}=\mathcal{F}_{0}{ }^{4}$ The function $s$ can be interpreted as coming from two different functions $s_{1}, s_{2}: \mathcal{F} \rightarrow \mathbb{R}_{+} \cup\{0\}$ (notice that they do not take negative values) which represent the stakes of bets that are bought and sold, respectively. That is, the agent is assumed to:

- buy bets for every event $E \in \mathcal{F}_{0}$ with the stake $s_{1}(E)$, i.e. she is willing to pay $q(E) s_{1}(E)$ for a bet that pays $s_{1}(E)$ if $E$ happens and 0 otherwise,

- $\quad$ sell bets for every event $E \in \mathcal{F}_{0}$ with the stake $s_{2}(E)$, i.e. she is willing to take $q(E) s_{2}(E)$ for a bet that pays $s_{2}(E)$ if $E$ happens and 0 otherwise.

It is easy to compute that when $s(E)$ is then defined as the difference $s_{1}(E)-s_{2}(E)$, then for any $w$ the value of the agent's profit with respect to an event $E$ is $s(E)(1-q(E))$ if $w \in E$ and $-s(E) q(E)$ otherwise. A Dutch Book is then defined as a series of bets (or, equivalently, a series of stakes) where the better's loss is guaranteed regardless of the choice of world $w$. In such a case we would alsy say that the function $s$ (together with the space $\langle W, \mathcal{F}, q\rangle$ ) constitutes a Dutch Book. Lastly, let us note that in our definition $\mathcal{F}_{0}$ is finite, since we only consider finite series of bets (even if the sample space is infinite).

The Reader may doubt whether the procedure to check whether the agent loses money "whatever happens", which we mentioned before giving the definition of a Dutch Book, can be simply applied in nonclassical cases, where the propositions may e.g. concern the outcomes of incompatible measurements: all bets in such cases should be conditional on which measurement is to be conducted. If so, then, to cut a long story short, we believe the Reader is right. In the next section we study the

\footnotetext{
${ }^{4}$ Observe that if we consider a bet that is neither bought nor sold, then we can just set its stake to 0 .
} 
fate of Feintzeig's project if we use the notions employed by him and the "classical" Dutch Book idea which he endorses, that is, the idea captured in Definition 1; then, in Section 4, we will use an alternative notion of a Dutch Book which seems to be more appropriate, and investigate how the project fares.

\section{Feintzeig's proposal}

Feintzeig uses in his theorems the notion of a "generalized probability space" in a version proposed by Gudder (1988). ${ }^{5}$ We need to quote the needed definitions here:

Definition 2 ( $\sigma$-additive class) A $\sigma$-additive class $\mathcal{F}$ on a set $W$ is a non-empty set of subsets of $W$ such that

1. for all $A \subseteq W$, if $A \in \mathcal{F}$, then $(W \backslash A) \in \mathcal{F}$; and

2. for all $\left\{A_{i}\right\}_{i \in \mathbb{N}} \subseteq \mathcal{F}$, if $A_{i} \cap A_{j}=\emptyset$ for any $i \neq j$, then $\cup_{i \in \mathbb{N}} A_{i} \in \mathcal{F}$.

Notice the "if" in point 2. of the definition: for some collections of subsets of $W$ their union might be excluded from $\mathcal{F}$.

Definition 3 (Generalized Probability Space) A generalized probability space is a triple $(W, \mathcal{F}, \mu)$, where $W$ is a non-empty set, $\mathcal{F}$ is a $\sigma$-additive class of subsets of $W$, and $\mu: \mathcal{F} \rightarrow \mathbb{R}$ is a real valued function such that

1. $\mu(W)=1$;

2. for any $A \in \mathcal{F} \mu(A) \geq 0$; and

3. if $A_{i} \in \mathcal{F}$ for any $i=1,2, \ldots$, and whenever $i \neq j, A_{i} \cap A_{j}=\emptyset$, then $\mu\left(\cup A_{i}\right)=\sum_{i} A_{i}$.

In Section 4 of his paper Feintzeig studies conditions which guarantee the existence of a classical extension of a given space. Suppose a nonclassical generalized probability space modelling some phenomena has a classical extension. This means that to capture these phenomena one could have used purely classical tools from the start, and the supposed nonclassicality of the phenomena results just from the initial description. The relevant notion of extension, going back again to Gudder, is as follows:

Definition 4 (Classical Probability Space Extension) A generalized probability space $(W, \mathcal{F}, \mu)$ has a classical probability space extension iff there is a classical probability space $\left(W, \mathcal{F}^{\prime}, \mu^{\prime}\right)$ such that $\mathcal{F} \subseteq \mathcal{F}^{\prime}$ and if $A \in \mathcal{F}$, then $\mu(A)=\mu^{\prime}(A)$.

\footnotetext{
${ }^{5}$ The definition of a " $\sigma$-additive class", a notion required for defining a generalized probability space, is on p. 913 of Feintzeig (2015) only an incomplete quote from Gudder: it requires closure just under finite disjoint unions, which is unexpected given the standard connotation of the " $\sigma-$ " prefix, that is, a connection with countable infinity. Fortunately this has no consequence for the validity of the theorems, because the structures used in the paper are finite. Nevertheless, we want to point this out here, because if the results are to be transferred to infinite examples this might become significant.
} 
Notice that the base set $W$ stays the same, and the connection between the two event classes $\mathcal{F}$ and $\mathcal{F}^{\prime}$ is just inclusion (not e.g. "being a subalgebra", which could be more natural for some).

We take a part of Feintzeig's message to be that generalized probability spaces of real use in philosophy of (quantum) physics should satisfy two desiderata:

1. they should not have classical extensions, i.e. they should describe inherently nonclassical phenomena;

2. they should avoid a certain form of (probabilistic) irrationality, i.e. they should not be Dutch-Bookable.

If one was to seek a justification for these desiderata (even though Feintzeig does not do so, an in this paper we are rather trying to explore their consequences), we would suggest the following route. If desideratum 1 above is not met, then, as already mentioned, the phenomena modelled by the generalized probability space could have been captured by a classical probability space from the start. That this is impossible for quantum phenomena has been argued e.g. by Suppes (1966). If desideratum 2 fails, then the space's probabilities cannot correspond to rational degrees of belief, and, in particular, to degrees of belief of a rational experimenter, and so cannot model a quantum experiment conducted by someone who believes in probabilities as offered by quantum mechanics.

It is not easy to formulate simple conditions referring directly to algebraic and probabilistic relations between events in a generalized probability space which would imply its having a classical extension. Feintzeig thinks a plausible one is that of finite subadditivity (labelled in his paper as “(**)"):

Definition 5 (Finite subadditivity) For a given (generalized) probability space $(W, \mathcal{F}, \mu)$, the measure $\mu$ is finitely subadditive iff for any finite collection of sets $\left\{A_{i}\right\}_{i=1, \ldots, n}$ such that $\mu\left(\bigcup_{i=1}^{n} A_{i}\right)$ is defined and for all $i \leq n$ the measure $\mu\left(A_{i}\right)$ is defined it holds that:

$$
\mu\left(\bigcup_{i=1}^{n} A_{i}\right) \leq \sum_{i=1}^{n} \mu\left(A_{i}\right) .
$$

In such a case we will also say that the space $(W, \mathcal{F}, \mu)$ satisfies finite subadditivity.

Feintzeig conjectures on p. 920 that all generalized probability spaces satisfying finite subadditivity have classical probability space extensions, suggesting also (on the same page) that finite subadditivity makes the space not susceptible to a Dutch Book. ${ }^{6}$ Unfortunately both these suggestions are false. We have the following theorem:

Theorem 1 There are (both finite and infinite) ${ }^{7}$ generalized probability spaces $(W, \mathcal{F}, \mu)$ satisfying finite subadditivity which do not have any classical extension.

\footnotetext{
${ }^{6}$ Remember that in this section we are following Feintzeig's idea regarding how Dutch Books should work in generalized probability spaces, which we hope to have captured in our Definition 1, p. 4.

${ }^{7}$ By "infinite" we mean here "such that both the base set $W$ and event class $\mathcal{F}$ are infinite", whereas by "finite" we mean a space with the base set being finite.
} 
and also an example of a Dutch Book on a space which satisfies finite subadditivity. See the Appendix for the proofs of both the finite and infinite cases; we also show just a single counterexample in the finite case; however, on that basis it is easy to prove that for any natural number $n \geq 6$ there is a generalized probability space $(W, \mathcal{F}, \mu)$, where $\operatorname{card}(W)=n$, which satisfies finite subadditivity but does not have any classical extension.

We now know that finite subadditivity does not force the existence of a classical extension, but also that it does not rule out susceptibility to a Dutch Book. Our next theorem will show that this behaviour is general: any condition we can impose on finite generalized propability spaces which does not imply classical extendability will also not imply immunity to Dutch Books. We show that the two bullet-point conditions from p. 7 are not jointly satisfiable by finite generalized probability spaces:

Theorem 2 A finite generalized probability space is Dutch-Bookable if and only if it does not possess a classical extension.

For the proof of the theorem, see Section A3 in the Appendix, p. 20. Interestingly, the theorem is false for spaces with an infinite base set.

Theorem 3 There exists an infinite generalized probability space which does not possess a classical extension and is not Dutch-Bookable.

Even though Theorem 3 shows that it is possible to meet Feintzeig's two desiderata using infinite generalized probability spaces, we would not want this to suggest that to represent quantum phenomena one should use such spaces. First, from inspecting the proof the Reader will see that to meet the desiderata such a space needs to be very carefully constructed in a way which does not seem to be related in any way to quantum phenomena. Second, there is a way of modelling such phenomena without necessarily using infinite sets, which we will discuss in the next section.

We have thus shown that the project of finding finite generalized probability spaces admitting no classical extensions and at the same time being not susceptible to a Dutch Book is unrealisable if we think of Dutch Books following (Feintzeig 2015). In the next section we will explore another option.

\section{Dutch Books in lattice-theoretic probability spaces}

Theorem 2 invites two responses. The first one would be that probabilities captured by generalized nonclassical probability spaces simply should not be understood as rational degrees of belief. This is certainly too hasty, but maybe with some merit: at this point we could say, for example, that the only promising way of thinking in that direction is to consider those probabilities as rational conditional degrees of belief. The second one is that the understanding of Dutch Books as defined by Definition 1 - which is implicit in Feintzeig's paper-has to be modified when we switch to a 
nonclassical setting. Again, a set of bets is a Dutch Book if the agent ends up with a loss "whatever turns out to be the case". What does the expression in scare quotes mean? Feintzeig uses the "classical" reading as captured by our Definition 1: we go through all the elements of the base set, and for each of those we take all propositions containing it to be true, and the others as false; we then count the money. This is certainly fine in classical cases. But in genuinely nonclassical scenarios perhaps the notion of a Dutch Book should be modified.

For example, consider a space $(W, \mathcal{F}, \mu)$ such that $A, B \in \mathcal{F}, A \cap B=\{x\} \notin \mathcal{F}$. When checking whether a given series of bets is a Dutch Book we eventually arrive at $x$. Do we take both $A$ and $B$ to be true in this case? Feintzeig would do so, and of course to discuss his proposal we have been so far doing the same in this paper. But if originally $A$ and $B$ were supposed to correspond to incompatible measurements, this is exactly what one should not do if one were willing to keep the model in accordance with the demands of quantum theory.

A change in formalism may also be helpful. While the notion of a generalized probability space defined above, introduced by Suppes (1966) and subsequently modified by Gudder (1969), has its adherents, some physicists and philosophers of physics (e.g. Pitowsky 2006) are content with employing as probability-bearers elements of structures more closely related to the formalism of quantum mechanics. In particular, the idea_-going back to Von Neumann (1932) - is (roughly) that in a given experimental seetting each set of possible values of some observable is to be identified with a family of orthogonal projections in a Hilbert space; all possible results of measurements to be conducted in a given run of the experiment correspond to the lattice of closed subspaces of that Hilbert space. The particulars of this idea have varied through the decades; for a summary see the beginning sections of Rédei and Summers (2007), and for a more abstract setting, which we will use here, see Rédei (1998). The main idea we want to point to is that the aforementioned lattice is an orthomodular lattice:

Definition 6 (Orthomodular lattice, orthogonality) An orthocomplemented lattice is an orthomodular lattice if for any its elements $e$ and $f$ the following holds:

$$
\text { if } e \leq f \text {, then } f=e \vee\left(e^{\perp} \wedge f\right) \text {. }
$$

Two elements $e$ and $f$ of an orthomodular lattice are called orthogonal if $e \leq f^{\perp}$.

Using these two concepts we can now define the other notion of a probability space we wish to discuss:

Definition 7 (Lattice-Theoretic Probability Space) A lattice-theoretic probability space is a tuple $(L, \mu)$, where $L$ is an orthomodular lattice and $\mu$ is an additive state on $L$, that is, is a map from $L$ to the $[0,1]$ segment of the reals satisfying the following:

1. $\mu\left(\mathbf{1}_{L}\right)=1$;

2. for any $E \subset L$ such that $E$ consists of mutually orthogonal elements, if $\vee E$ exists, then $P(\vee E)=\sum_{e \in E} \mu(e)$. 
Notice that since Boolean algebras are orthomodular lattices, then of course the event classes of classical probability spaces considered together with their measures are lattice-theoretic probability spaces. An important way of looking at this is the following:

a state on an orthomodular lattice restricted to a maximal Boolean sublattice is a classical probability function.

The idea naturally arises when we think of families of possible values of measurements which cannot be performed together: each measurement has its own Boolean algebra of results, and these algebras are "pasted together" to obtain an orthomodular lattice (the specifics of that operation will not be needed here; see Greechie 1968). But if we restrict our attention to just a single measurement, what we "see" is just a classical probability space.

It seems that when Dutch Books, or in fact any bets at all, have been discussed in philosophy of physics, the underlying structure was that of a lattice-theoretic probability space, even if it was not explicitly mentioned, because the given approached dealt directly with the Hilbert space formalism (as in the case of the various papers on "quantum bayesianism", e.g. Caves et al. 2002) or spoke about collections of Boolean algebras associated with particular observables (as in the case of e.g. Pitowsky 2003).

How should we think of betting in the context of lattice-theoretic probability spaces, if we restrict our attention to those of interest to philosophers of quantum physics, namely those which result from pasting together Boolean "blocks" corresponding to various measurements? (Assume we keep this restriction from now on.) Pitowsky (2006) (section 3.2) contains a particularly clear view of this, which we will quote here:

"Our gambles will (...) be finite and consist of four steps:

- A single physical system is prepared by a method known to everybody.

- A finite set $M$ of incompatible measurements, each with a finite number of possible outcomes, is announced by the bookie. The agent is asked to place bets on the possible outcomes of each one of them.

- One of the measurements in the set $M$ is chosen by the bookie and the money placed on all other measurements is promptly returned to the agent.

- The chosen measurement is performed and the agent gains or loses in accordance with his bet on that measurement."

In the lattice-theoretic context the $M$ from the above quote will be a Boolean "block" (i.e. maximal Boolean sublattice) of the orthomodular lattice. It is clear that the bets to be considered are conditional bets, just like the once used in diachronic Dutch Book arguments in formal epistemology (see Bradley (2015) for an introduction). They are only "in place" if the particular measurement is performed in the given run of the experiment; they are void otherwise.

What would a Dutch Book look like in a lattice-theoretic probability space? It would be a finite set of bets, guaranteeing the agent's loss, which we could of course split (in possibly more than one way) into sets of bets concerning propositions from separate Boolean "blocks" of the lattice. But this has to mean that at least one of these smaller sets of bets would guarantee a loss of the agent, therefore the state 
on the lattice, when restricted to a particular Boolean sublattice, would not be a classical probability function (by the classical Dutch Book theorems). This would contradict (*).

We see, therefore, that lattice-theoretic probability spaces are not susceptible to Dutch Books. This is as it should be if nonclassical probability is to be a generalization of classical probability. This means that in the context of lattice-theoretic probability spaces half of the project from p. 7 disappears: what remains is the task of finding spaces which do not possess a classical extension.

Here, though, a different issue appears. Namely: it is decidedly non-trivial what an extension of a lattice-theoretic probability space is. Some (see e.g. Hofer-Szabó et al. (2000), and numerous later papers by the same authors) think that an extension of a lattice-theoretic probability space $(L, \mu)$ is a space $\left(L^{\prime}, \mu^{\prime}\right)$ such that there is an embedding $h: L \rightarrow L^{\prime}$ (that is, an injection which preserves all lattice operations) such that for all $W \in L, \mu(W)=\mu^{\prime}(h(W))$. That is, the "new" lattice of events contains the "old" one as a sublattice, and the "new" state preserves the "old" probabilities. Notice, though, that if we use this notion of extension, then the question of existence of a classical extension of a nonclassical lattice-theoretic probability space is trivial: such an extension does not exist, since there is no embedding (in the above sense) of a nondistributive orthomodular lattice into a distributive one.

Perhaps, then, a different notion of extension is needed. So far we have not been able to obtain an intuitive one which would not trivialise the above task. We leave this, then, to a future paper.

\section{Conclusion}

We have studied bets regarding elements of two types of nonclassical probability spaces in the context of the following project: to find nonclassical probability spaces not possessing a classical extension which would not be susceptible to a Dutch Book. Our Theorem 2 shows that the project fails regarding generalized probability spaces: no such spaces satisfy the two criteria, at least no finite ones, and these seem to be of the main interest to the authors in the field. We have also argued that if we move to lattice-theoretic probability spaces, then one part of the project is completed trivially, since no such spaces are Dutch-Bookable, but the other part either trivially fails, or it is unclear how to proceed to pursue it. We hope some interesting notion of extension can be worked out for the lattice-theoretic case.

Acknowledgments We would like to thank Balázs Gyenis and Tomasz Placek for insightful discussions. LW's work on the paper was a part of the joint project of the Polish and Hungarian Academies of Science "Probability, Causality and Determinism".

LW's and MTG's work was supported by Tomasz Placek's grant "Modalities, probability and Bell Theorems" as a part of the "MISTRZ" program of the Foundation for Polish Science, as well as the NCN "Sonata" grant 2015/17/D/HS1/01912 "Epistemic inaccuracy and degrees of belief".

Open Access This article is distributed under the terms of the Creative Commons Attribution 4.0 International License (http://creativecommons.org/licenses/by/4.0/), which permits unrestricted use, distribution, and reproduction in any medium, provided you give appropriate credit to the original author(s) and the source, provide a link to the Creative Commons license, and indicate if changes were made. 


\section{Appendix}

\section{A1}

Theorem 1 (p. 10:) There are (both finite and infinite) generalized probability spaces $(W, \mathcal{F}, \mu)$ satisfying finite subadditivity which do not have any classical extension.

Proof We first show a finite counterexample to Feintzeig's conjecture, using a space similar to the one employed in Prather (1980) (p. 1), but used there with different goals in mind. Let $W=\{1,2,3,4,5,6\}$. Let $\mathcal{F}=\{A \subseteq W: \operatorname{card}(A)=3\} \cup\{W\} \cup \emptyset$ and let $\mu: \mathcal{F} \rightarrow[0,1]$ be the following:

- $\mu(W)=1, \mu(\emptyset)=0$,

- $\mu(\{1,3,5\})=\frac{3}{4}, \mu(\{2,4,6\})=\frac{1}{4}$, and

- $\mu(A)=\frac{1}{2}$ for any $A \in \mathcal{F}$ different from all of the above.

It is easy to see that $\mathcal{F}$ is a $\sigma$-additive class and that $\mu$ is a finitely subadditive probabilistic measure on $\mathcal{F}$.

Suppose, for a contradiction, that there is a $\sigma$-algebra ( $\sigma$-field) of sets $\mathcal{F}^{\prime} \subseteq \mathcal{P}(W)$ such that $\mathcal{F} \subseteq \mathcal{F}^{\prime}$ and a probabilistic measure $\mu^{\prime}: \mathcal{F}^{\prime} \rightarrow[0,1]$ such that $\forall A \in$ $\mathcal{F} \mu^{\prime}(A)=\mu(A)$, i.e. $\mu^{\prime}\lceil\mathcal{F}=\mu$.

First, observe that for any $x \in W$ the $\sigma$-algebra $\mathcal{F}^{\prime}$ contains a singleton $\{x\}$. This is because for any pairwise different $a_{1}, \ldots a_{5} \in W$ such that $a_{i} \neq x$ the sets $\left\{a_{1}, a_{2}, a_{3}\right\}$ and $\left\{a_{3}, a_{4}, a_{5}\right\}$ belong to $\mathcal{F}$, and so to $\mathcal{F}^{\prime}$; therefore $\left\{a_{1}, \ldots, a_{5}\right\}$ is in $\mathcal{F}^{\prime}$, since $\mathcal{F}^{\prime}$ is a $\sigma$-algebra. Hence, as $\mathcal{F}^{\prime}$ is closed under complements, $\{x\} \in \mathcal{F}^{\prime}$. Thus, $\mathcal{F}^{\prime}$, as containing all the singletons over $W$, is actually unique, namely $\mathcal{P}(W)$. Now, by the fact that $\mu^{\prime}$ is a probabilistic measure we easily obtain that:

$$
\begin{aligned}
& \mu^{\prime}(\{1\})=\mu^{\prime}(\{3\})=\mu^{\prime}(\{5\}), \\
& \mu^{\prime}(\{2\})=\mu^{\prime}(\{4\})=\mu^{\prime}(\{6\}),
\end{aligned}
$$

since

$\mu^{\prime}(\{1,2,4\})=\mu^{\prime}(\{1\})+\mu^{\prime}(\{2\})+\mu^{\prime}(\{4\})=\mu^{\prime}(\{3\})+\mu^{\prime}(\{2\})+\mu^{\prime}(\{4\})=\mu^{\prime}(\{3,2,4\})$, so $\mu^{\prime}(\{1\})=\mu^{\prime}(\{3\})$, and also

$\mu^{\prime}(\{5,2,4\})=\mu^{\prime}(\{5\})+\mu^{\prime}(\{2\})+\mu^{\prime}(\{4\})=\mu^{\prime}(\{3\})+\mu^{\prime}(\{2\})+\mu^{\prime}(\{4\})=\mu^{\prime}(\{3,2,4\})$, so $\mu^{\prime}(\{3\})=\mu^{\prime}(\{5\})$. We also have

$\mu^{\prime}(\{2,3,5\})=\mu^{\prime}(\{2\})+\mu^{\prime}(\{3\})+\mu^{\prime}(\{5\})=\mu^{\prime}(\{4\})+\mu^{\prime}(\{3\})+\mu^{\prime}(\{5\})=\mu^{\prime}(\{3,4,5\})$, so $\mu^{\prime}(\{2\})=\mu^{\prime}(\{4\})$, and also

$\mu^{\prime}(\{3,4,5\})=\mu^{\prime}(\{4\})+\mu^{\prime}(\{3\})+\mu^{\prime}(\{5\})=\mu^{\prime}(\{6\})+\mu^{\prime}(\{3\})+\mu^{\prime}(\{5\})=\mu^{\prime}(\{6,4,5\})$, so $\mu^{\prime}(\{4\})=\mu^{\prime}(\{6\})$. Thus, for any $x=1,3,5$ and any $y=2,4,6$ :

$$
\mu^{\prime}(\{x\})=\mu^{\prime}(\{y\})+\frac{1}{4},
$$


which is because $\mu^{\prime}$ extends $\mu$ and we have e.g.

$$
\begin{gathered}
\mu^{\prime}(\{1\})+\mu^{\prime}(\{3\})+\mu^{\prime}(\{5\})=\mu^{\prime}(\{1,3,5\})=\frac{3}{4} \\
=\frac{1}{2}+\frac{1}{4}=\mu^{\prime}(\{2,3,5\})+\frac{1}{4}=\mu^{\prime}(\{2\})+\mu^{\prime}(\{3\})+\mu^{\prime}(\{5\})+\frac{1}{4},
\end{gathered}
$$

so the equation holds for $x=1$ and $y=2$ and the same goes for any other choice of $x=1,3,5$ and any $y=2,4,6$. Since $\mu^{\prime}$ extends $\mu$ and $\mu(\{2,4,6\})=\frac{1}{4}$ we have:

$$
\mu^{\prime}(\{2\})=\mu^{\prime}(\{4\})=\mu^{\prime}(\{6\})=\frac{1}{12} .
$$

But then, for any $x=1,3,5$ we have:

$$
\mu^{\prime}(\{1\})=\mu^{\prime}(\{3\})=\mu^{\prime}(\{5\})=\frac{1}{3},
$$

and so

$$
\mu^{\prime}(\{1,3,5\})=1,
$$

which is impossible, since (by our reductio assumption) $\mu^{\prime}(\{1,3,5\})=$ $=\mu(\{1,3,5\})=\frac{3}{4}$ which ends the proof in the finite case.

To see that the theorem also holds in the infinite, let $W=\mathbb{N}$, let

$$
A=\{0,2,4,6, \ldots\}
$$

and

$$
\bar{A}=\{1,3,5,7, \ldots\},
$$

and for all odd natural numbers $n$ put

$$
B_{n}=\{0\} \cup \bar{A} \backslash\{n\},
$$

i.e. $B_{1}=\{0,3,5,7,9 \ldots\}, \overline{B_{1}}=\{1,2,4,6, \ldots\}, B_{3}=\{0,1,5,7,9 \ldots\}, \overline{B_{3}}=$ $\{3,2,4,6,8, \ldots\}$ and so on.

Let

$\mathcal{F}=\left\{B_{n} \subseteq W: \exists k n=2 k+1\right\} \cup\left\{\overline{B_{n}} \subseteq W: \exists k n=2 k+1\right\} \cup\{W\} \cup \emptyset \cup A \cup \bar{A}$ and let $\mu: \mathcal{F} \rightarrow[0,1]$ be the following:

$$
\begin{aligned}
& \mu(W)=1, \mu(\emptyset)=0, \\
& \mu(A)=\frac{1}{4}, \mu(\bar{A})=\frac{3}{4},
\end{aligned}
$$

and

$$
\mu\left(B_{n}\right)=\mu\left(\overline{B_{n}}\right)=\frac{1}{2}
$$

for any $B_{n} \in \mathcal{F}$ different then one of the set above.

It is easy to see that $\mathcal{F}$ is a $\sigma$-class, $\mu$ is a probabilistic measure on $\mathcal{F}$ and $\mu$ satisfies the subadditivity of the measure. It is so, since apart from the sets that are pairwise complement, all pairs of non-trivial elements of the $\sigma$-class have nonempty intersections and no pair of nontrivial sets that are not pairwise complement sums up to the entire set $\mathbb{N}$.

Suppose, for a contradiction, that there is a $\sigma$-algebra of sets $\mathcal{F}^{\prime} \subseteq \mathcal{P}(W)$ such that $\mathcal{F} \subseteq \mathcal{F}^{\prime}$ and a probabilistic measure $\mu^{\prime}: \mathcal{F}^{\prime} \rightarrow[0,1]$ such that $\forall A \in \mathcal{F} \mu^{\prime}(A)=$ $\mu(A)$, i.e. $\mu^{\prime}\lceil\mathcal{F}=\mu$. 
First, observe that $\{0\} \in \mathcal{F}^{\prime}$. Indeed, $\bar{A} \cup \overline{B_{1}}=\mathbb{N} \backslash\{0\} \in \mathcal{F}^{\prime}$, by the fact that $\mathcal{F}^{\prime}$ is a $\sigma$-algebra. Also, for any odd $n \in \mathbb{N}$ it is the case that $\{n\} \in \mathcal{F}^{\prime}$, since $A \cup B_{n}=$ $\mathbb{N} \backslash\{n\} \in \mathcal{F}^{\prime}$, by the very same fact again.

Hence, the set $A \backslash\{0\}$ also has to be in $\mathcal{F}^{\prime}$.

Thus, we have:

$$
\mu^{\prime}(\{0\})+\mu^{\prime}(A \backslash\{0\})=\mu^{\prime}(A)=\mu(A)=\frac{1}{4},
$$

and:

$$
\mu^{\prime}(\{n\})+\mu^{\prime}(A \backslash\{0\})=\mu^{\prime}\left(\overline{B_{n}}\right)=\mu\left(\overline{B_{n}}\right)=\frac{1}{2} .
$$

Therefore, for any odd $n \in \mathbb{N}$, we have

$$
\mu^{\prime}(\{n\})=\mu^{\prime}(\{0\})+\frac{1}{4} .
$$

But then, the measure $\mu^{\prime}$ of the set $A$ of odd numbers is infinite (as an infinite sum of sets with a constant positive measure) which is impossible.

Here we can see that it would be enough to take $B_{1}, B_{3}, B_{5}$ and $B_{7}$ to see that then $\mu^{\prime}(A) \geq 4 \cdot \frac{1}{4}$ which is already a contradiction.

\section{A2}

A Dutch Book on a space which satisfies finite subadditivity Here we point out that, contrary to Feintzeig's suggestion, finite subadditivity does not guarantee nonsusceptibility to a Dutch Book. This of course follows directly from Theorems 1 and 2 ; the following is just a concrete illustration.

The following are the assumptions we make for this and next section of the Appendix, in which we continue to discuss Dutch Books:

- Assume for convenience each bet pays $\$ 1$ when won (and \$0 when lost).

- An agent considers buying or selling a bet on a proposition (event) $A$ for the price $x$ to be fair iff his degree of belief in $A$ is $x$.

- A Dutch Book against the given agent is a collection of bets (sold and/or bought) such that the agent considers each bet in the collection to be fair, but which, taken together, guarantee his loss.

- We will call a generalized probability space "Dutch-Bookable" (saying also that its probability function "permits a Dutch Book") iff, if the probabilities are thought of as degrees of belief for some agent, there exists a Dutch Book against that agent involving only bets on propositions (events) belonging to the class of events of that probability space.

Consider the space defined in Section A1 and an agent setting the degrees of his belief according to the measure $\mu$ of this space. The agent will agree that the following procedure has him buy and sell fair bets:

1. he buys:

- 1 bet for $\{1,3,5\}$ for $\$ 0.75$; 
2. he sells:

- 3 bets for $\{2,4,6\}$ for $\$ 0.75$ together (i.e. if $\{2,4,6\}$ turns out to be true, he has to pay $\$ 3$ to the person who bought the bets; each bet costs $\$ 0.25$ since he believes the event has probability $\left.\frac{1}{4}\right)$;

- 1 bet for $\{2,3,5\}$ for $\$ 0.50$;

- 3 bets for $\{1,2,3\}$ for $\$ 1.50$ together;

- 3 bets for $\{1,4,5\}$ for $\$ 1.50$ together;

- 3 bets for $\{3,5,6\}$ for $\$ 1.50$ together;

- 1 bet for $\{1,3,4\}$ for $\$ 0.50$.

After trading the bets he has $\$+5.50$, but whatever the outcome, he ends up with at best $\$-0.50$, thus this is a Dutch Book against him. And so the space is DutchBookable. $^{8}$

\section{A3}

Theorem 2 (p. 10:) A finite generalized probability space is Dutch-Bookable if and only if it does not possess a classical extension.

Proof We will use the well-known fact by de Finetti that classical probability spaces are not Dutch-Bookable. We will also use the following fact from Paris (2001) ("SL" is the set of sentences of a finite propositional language $L$, composed using standard connectives $\vee, \wedge, \neg$ and $\rightarrow-$ each such $S L$ can be thought of a set of events over some universe $L$ ):

Fact 1 (Paris 2001, p. 5) Let $B$ be a partial function from $S L$ into [0, 1]. Then $B$ does not permit a Dutch Book if and only if $B$ can be extended to a full function from $S L$ into $[0,1]$ which does not permit a Dutch Book, i.e. which is a classical probability function.

The arrows below refer to the directions of the implications in Theorem 2.

$(\Rightarrow)$ Suppose a generalized probability space $(W, \mathcal{F}, \mu)$ is Dutchbookable. Suppose also, for reductio, that it does possess a classical extension $\left(W^{\prime}, \mathcal{F}^{\prime}, \mu^{\prime}\right)$. Since $\mathcal{F} \subseteq \mathcal{F}^{\prime}$, then $\left(W^{\prime}, \mathcal{F}^{\prime}, \mu^{\prime}\right)$ is also Dutch-Bookable. But $\left(W^{\prime}, \mathcal{F}^{\prime}, \mu^{\prime}\right)$ is a classical probability space, and so, by the classical result of de Finetti, is not Dutch-Bookable. Contradiction.

$(\Leftarrow)$ We show the counterpositive. Suppose the (finite, as assumed) generalized probability space $(W, \mathcal{F}, \mu)$ is non-classical (i.e. $\mathcal{F}$ is not a Boolean algebra of subsets of $W$ ) and not Dutch-bookable. Notice that $\mathcal{F}$ is a proper subset of some Boolean algebra of subsets of $W$; that algebra, in turn, is a subalgebra of some finite free Boolean algebra. Therefore, for some finite propositional language $L, \mu$ can be

\footnotetext{
${ }^{8}$ It is easy to create an example of an infinite space satisfying finite subadditivity but falling prey to a Dutch Book; we omit it for brevity.
} 
thought of as a partial function from $S L$ to $[0,1]$. Since by assumption $(W, \mathcal{F}, \mu)$ is not Dutch-Bookable, by Fact 1 we infer that $\mu$ can be extended to a full function $\mu^{\prime}$ from $S L$ to $[0,1]$ which does not permit a Dutch Book, that is, that there is a Boolean algebra $\mathcal{F}^{\prime}$ of subsets of $W$ and a function $\mu$ such that $\left(W, \mathcal{F}^{\prime}, \mu^{\prime}\right)$ is a classical probability space extension of $(W, \mathcal{F}, \mu)$.

\section{A4}

Theorem 3 (p. 8:) There exists an infinite generalized probability space which does not possess a classical extension and is not Dutch-Bookable.

Proof Consider the set $W=\mathbb{Z} \backslash\{0\}=\mathbb{N}_{+} \cup \mathbb{N}_{-}$.

Define the following subsets of $W: A_{0}:=\{1,2,3,4, \ldots\}, A_{1}:=$ $\{-1,2,3,4, \ldots\}, A_{2}:=\{1,-2,3,4, \ldots\}, \ldots$

More generally, put:

$$
A_{n}:=\{1,2, \ldots, n-1,-n, n+1, \ldots\},
$$

Define the following $\sigma$-additive class:

$$
\mathcal{F}=\left\{A_{n} \subseteq W: n \in \omega\right\} \cup\left\{\overline{A_{n}} \subseteq W: n \in \omega\right\} \cup\{W, \emptyset\},
$$

and set a probabilistic measure $\mu: \mathcal{F} \rightarrow[0,1]$ as follows:

$$
\forall n \mu\left(A_{n}\right)=1, \mu(W)=1 .
$$

Observe that the space $(W, \mathcal{F}, \mu)$ has no classical extension. Indeed if there were a $\sigma$-algebra $\mathcal{F}^{\prime} \supset \mathcal{F}$ and a probabilistic measure $\mu^{\prime}: \mathcal{F}^{\prime} \rightarrow[0,1]$ such that $\mu^{\prime}: \uparrow \mathcal{F}=\mu$, then we would have:

$$
\mu^{\prime}\left(\bigcup_{n \in \mathbb{N}} \overline{A_{n}}\right)=\mu^{\prime}(W)=\mu(W)=1,
$$

but

$$
\sum_{n \in \mathbb{N}} \mu^{\prime}\left(\overline{A_{n}}\right)=\sum_{n \in \mathbb{N}} \mu\left(\overline{A_{n}}\right)=0
$$

which would obviously contradict additivity.

However, we claim that there is no Dutch Book on this space. We even prove a stronger claim, namely that: for any (finite) set of bets $Z$ on the space $(W, \mathcal{F}, \mu)$, there exists an $n_{0}>0$ such that for all $n>n_{0} U_{Z}(n) \geq 0$, where $U_{Z}(n)$ denotes the agent's payoff with the set of bets $Z$ and the result $n$.

To see this, take any bet $Z$. The list of all bets bought in the set of bets $Z$ is the following:

- $b_{1}$ bets on the sets with measure of type

$$
A_{k}=\{1,2, \ldots, k-1,-k, k+1, \ldots\} \text {, i.e. such that } \mu\left(A_{k}\right)=1 \text {, }
$$

- $b_{2}$ bets on the sets of type

$$
\overline{A_{l}}=\{-1,-2, \ldots,-l+1, l,-l-1, \ldots\} \text {, i.e. such that } \mu\left(\overline{A_{l}}\right)=0 \text {. }
$$

The list of all bets sold in the set of bets $Z$ is the following: 
- $c_{1}$ bets on the sets of type

$A_{m}=\{1,2, \ldots, m-1,-m, m+1, \ldots\}$, i.e. such that $\mu\left(A_{m}\right)=1$,

- $c_{2}$ bets on the sets of type

$\overline{A_{p}}=\{-1,-2, \ldots,-p+1, p,-p-1, \ldots\}$, i.e. such that $\mu\left(\overline{A_{p}}\right)=0$.

So, the set of bets $Z$ can be presented as follows:

$$
Z=\langle\underbrace{A_{k_{1}}, \ldots, A_{k_{b_{1}}}, \overline{A_{l_{1}}}, \ldots, \overline{A_{l_{b_{2}}}}}_{\text {bought }}, \underbrace{\left.A_{m_{1}}, \ldots A_{m_{c_{1}}}, \overline{A_{p_{1}}}, \ldots, \overline{A_{p_{c_{2}}}}\right\rangle}_{\text {sold }} .
$$

Observe now that the agent has to pay $b_{1}$ for the bought bets on sets with measure 1 (i.e. of type $A_{k}$ ) and receives $c_{1}$ for the sold bets on sets with measure 1 (i.e. of type $\left.A_{m}\right)$ and neither pays nor receives anything for any other bets (i.e. of type $\overline{A_{l}}$ or of type $\overline{A_{p}}$ ).

Now put

$$
n_{0}:=1+\max \left\{k_{1}, \ldots, k_{b_{1}}, l_{1}, \ldots, l_{b_{2}}, m_{1}, \ldots, m_{c_{1}}, p_{1}, \ldots, p_{c_{2}}\right\} .
$$

If the outcome of the bet is any $n \geq n_{0}$, by the definitions of the elements of our space, the agent:

- receives $b_{1}$ for the bets of type $A_{k}$ he bought, since for every $n \geq n_{0}$ and each $i \leq b_{1}$ it holds that $n \in A_{k_{i}}$;

- $\quad$ pays $c_{1}$ for the bets of type $A_{m}$ he sold, since for every $n \geq n_{0}$ and each $j \leq c_{1}$ it holds that $n \in A_{m_{j}}$;

and

- neither pays nor obtains anything for the bets on any other sets (i.e. of type $\overline{A_{l}}$ or of type $\overline{A_{p}}$ ), since for every $n \geq n_{0}$ and for each $i \leq b_{2}$ and each $j \leq c_{2}$ we have that $n \notin \overline{A_{l_{i}}}$ and $n \notin \overline{A_{p_{j}}}$.

Thus, for every $n \geq n_{0}$ the net $U_{Z}(n)=0$.

\section{References}

Bradley, D. (2015). A critical introduction to formal epistemology: Bloomsbury Publishing.

Caves, C.M., Fuchs, C.A., \& Schack, R. (2002). Quantum probabilities as Bayesian probabilities. Physical Review A, 65(2), 022305.

Christensen, D. (2004). Putting logic in its place. Formal constraints on rational belief. Oxford: Clarendon Press.

Feintzeig, B. (2015). Hidden variables and incompatible observables in quantum mechanics. The British Journal for the Philosophy of Science, 66(4), 905-927.

Fine, T.L. (1973). Theories of probability: Academic Press.

Greechie, R. (1968). On the structure of orthomodular lattices satisfying the chain condition. Journal of Combinatorial Theory, 4(3), 210-218.

Gudder, S.P. (1969). Quantum probability spaces. Proceedings of the American Mathematical Society, 21, 296-302.

Gudder, S.P. (1988). Quantum probability: Academic Press.

Hofer-Szabó, G., Rédei, M., \& Szabó, L.E. (2000). Common cause completability of classical and quantum probability spaces. International Journal of Theoretical Physics, 39(3), 913-919.

Kemeny, J.G. (1955). Fair bets and inductive probabilities. The Journal of Symbolic Logic, 20(3), 263-273.

Von Neumann, J. (1932). Mathematische Grundlagen der Quantenmechanik: Verlag von Julius Springer Berlin. 
Paris, J.B. (2001). A note on the Dutch Book method. In ISIPTA, Vol. 1.

Pitowsky, I. (2003). Betting on the outcomes of measurements: a Bayesian theory of quantum probability. Studies in History and Philosophy of Science Part B: Studies in History and Philosophy of Modern Physics, 34(3), 395-414.

Pitowsky, I. (2006). Quantum mechanics as a theory of probability. In Physical theory and its interpretation (pp. 213-240): Springer.

Prather, R.E. (1980). Generating the k-subsets of an n-set. American Mathematical Monthly, 87(9), 740743.

Ramsey, F.P. (1931). Truth and probability (1926). The foundations of mathematics and other logical essays, 156-198.

Rédei, M. (1998). Quantum logic in algebraic approach: Springer Science \& Business Media.

Rédei, M., \& Summers, S.J. (2007). Quantum probability theory. Studies in History and Philosophy of Science Part B: Studies in History and Philosophy of Modern Physics, 38(2), 390-417.

Suppes, P. (1966). The probabilistic argument for a non-classical logic of quantum mechanics. Philosophy of Science, 1(/2), 14-21.

Vineberg, S. (2011). Dutch Book arguments, In Zalta, E.N. (Ed.) The Stanford Encyclopedia of Philosophy: Summer 2011 edition. 